\title{
Effects of Varying Haptic Feedback on Driver Distraction During Vehicular Window Adjustment
}

\author{
John K. Holmen \\ The Electrical and Computer Engineering Department \\ Kettering University \\ Flint, United States \\ holm5073@kettering.edu
}

\author{
Mehrdad Hosseini Zadeh \\ The Electrical and Computer Engineering Department \\ Kettering University \\ Flint, United States \\ mzadeh@kettering.edu
}

\begin{abstract}
Haptic-enabled rotary control knobs are increasingly being integrated within vehicles to manage vehicular instrumentation. By doing so, driver safety and performance is increased as a result of the distraction reductions associated with such a system. The integration of window adjustment within such a vehicular instrumentation management system is examined through human factors studies for the purpose of reducing driver distraction. Additional focus is placed on examining the ability of haptic feedback alone to eliminate reliance on visual feedback when adjusting window height. Results indicate that eliminating such reliance is possible by means of a rotary control knob providing varying intermediate haptic feedback as the window is adjusted.
\end{abstract}

\section{Categories and Subject Descriptors}

H.5.2 [Information Interfaces and Presentation]: User Interfaces - Haptic I/O.

\section{General Terms}

Performance, Design, Human Factors.

\section{Keywords}

Varying intermediate haptic feedback, rotary control knob, window adjustment, window position, driver distraction.

\section{INTRODUCTION}

Examining vehicle interiors, current window adjustment systems rely heavily upon visual and auditory feedback to determine window height. By utilizing a haptic-enabled rotary control knob, it is possible for this reliance on visual feedback to be eliminated as the reliance shifts towards a combination of haptic and auditory feedback. Such a shift utilizes senses least used during driving (e.g. touch) and has the ability to improve driving and task performance [7, 9, 11]. These performance increases are attributed to both the reduction of errors and the time spent performing the task. Most importantly, such instrumentation control devices reduce the likelihood of an accident [9].

In recent years, several major automotive manufacturers have incorporated instrumentation control into a single rotary control knob $[1,2,4,6]$. Such a knob has the ability to provide a driver with quick and easy access to instrumentation functionality $[2,3$,

Copyright held by author(s)

AutomotiveUI'10, November 11-12, 2010, Pittsburgh, Pennsylvania ACM 978-1-4503-0437-5
$4,6,7,12]$. Furthermore, such a knob has the ability to effectively deliver information and cues to the driver when paired with haptic feedback [8]. As such, the primary focus of the driver is allowed to remain on driving, increasing the safety of the driver [7, 9]. Examples of such instrumentation control devices include the Multi Media Interface (MMI) offered by Audi [2], the iDrive control system offered by BMW [4], the COMAND control system offered by Mercedes Benz [6], and advanced control panel solutions offered by Visteon [6]. However, these devices do not incorporate both window adjustment and haptic feedback.

We designed a haptic-enabled rotary control knob in [10] for the purpose of selecting a window within a vehicle for adjustment. In this paper, we expand the previous investigation by now focusing on the adjustment of window height. Through the associated study, the possibility of removing driver reliance on visual feedback during window height adjustment is examined for the purpose of reducing driver distraction. This investigation is accomplished by utilizing the haptic-enabled rotary control knob and a supporting GUI for experimentation. Human factors studies were then conducted to examine the effects of integrating window height adjustment into an instrumentation control device such as the BMW iDrive control system. Using this device in conjunction with a driving simulator, the following hypothesis was tested:

$\mathbf{H}_{\mathbf{1}}$ : Adding varying intermediate haptic feedback to a rotary control knob allows drivers to accurately adjust window height while eliminating reliance on visual feedback.

To evaluate the validity of this hypothesis, a single study was conducted to examine the accuracy with which a driver could adjust window height when provided with only a haptic-enabled rotary control knob. Within this paper, the work related to this study will first be discussed. The experimental setup and procedure will then be discussed. Following this, the results of the study will be analyzed and further discussed. Lastly, concluding remarks and future work will be presented.

\section{RELATED WORK}

Considering the ability of haptic feedback to effectively deliver information and cues to a user [8], it has great potential to improve task performance in specific situations. The utilization of haptic feedback to improve user experience has been thoroughly investigated through a wide range of applications. Such applications include teleoperation [5], automotive, and even utilizing haptics within a doorknob to convey information [8].

Focusing on automotive applications, contributions made by Immersion Corporation are most relevant to the work presented. This corporation has partnered with BMW, Lexus, Mercedes- 
Benz, and Visteon to implement haptic feedback within vehicles $[4,6]$. With the exception of Lexus, each of these manufacturers has utilized a haptic-enabled rotary control knob to manage entertainment, navigation, and climate control systems. Ultimately, these systems have allowed users to cut glances time, reduce distractions, and improve safety while driving [6].

Furthermore, Immersion Corporation has conducted a study aimed at verifying the safety and performance benefits that drivers experience as a result of haptic feedback [9]. This study consisted of two experiments performed while driving within a virtual environment. The first experiment focused on the lookup of lowattention information while driving by requiring the user to locate and select a target on a static graphical image of a slider. The second experiment extended upon this by requiring the user to locate and select a target in two tiers.

The results of this study suggested that drivers utilizing "smart" instrumentation controls would be less likely to drive off the road. Additionally, results suggested that adding programmable touch sensations to instrument controls can improve operator performance in both the instrument task and a primary driving task. Most importantly, results suggested that simple haptic feedback increases driving safety by freeing attention for actual driving. While this work focuses on safety and performance benefits on a general level, current work aims to validate the benefits associated with utilizing varying durations of haptic feedback for the purpose of adjusting window height.

Additionally, a series of studies were conducted in [10] analyzing the benefits of utilizing visual feedback, haptic feedback, and both visual and haptic feedback for the purpose of window selection utilizing a haptic-enabled rotary control knob. Haptic feedback was generated in the form of a constant force of $0.83 \pm 0.01 \mathrm{~N}$ exerted on the subject's hand when transitioning between window boundaries (see Figure 1). Attention was also given to favoring the boundaries surrounding a specific window by using a $1.34 \pm 0.02 \mathrm{~N}$ force for differentiation. As a result of these studies, it was strongly confirmed that the addition of haptic feedback within a proposed centrally located window selection knob has the potential to increase driver performance by allowing focus to remain on the road. Favoring a window resulted in positive performance improvements compared to the non-favored case.

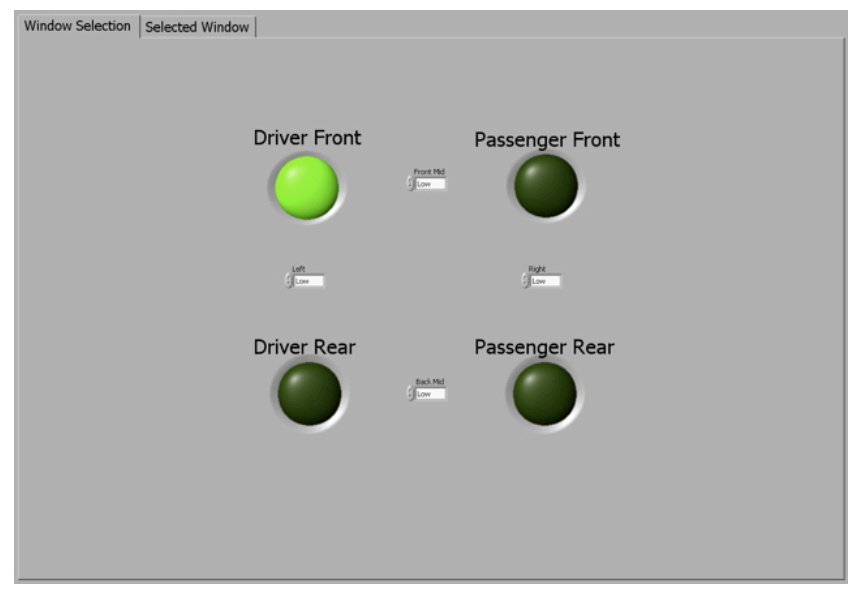

Figure 1. Window Select screen utilized within a previous study to monitor the current window selected and the haptic feedback level utilized during experimentation.
However, this comparison requires additional data to approach statistical significance. Current work expands upon this vehicular window control system by analyzing the adjustment of window height as opposed to selecting windows. Furthermore, focus is placed on exploring the potential benefits resulting from utilizing varying durations of force to indicate window height.

\section{EXPERIMENTAL SETUP AND PROCEDURE}

Experimentation was conducted with the assistance of a driving simulator to recreate the task of driving (see Figure 2). This was used to analyze window height adjustment performance while providing the subject with a primary driving task to focus on. The driving simulator consisted of a seat, force-feedback steering wheel, pedals for gas and brake, a display for the driving task, a display for the window adjustment task, and a haptic-enabled rotary control knob placed within the reach of the right hand. For the purpose of this experiment, the force-feedback capabilities of the steering wheel were used to add stiffness to the steering wheel. This was done to simulate the feel of power steering found in current vehicles. No additional forms of force-feedback were applied to the steering wheel during this experiment.

Focusing on the display used by the subject, this presented the subject with a virtual environment supporting the driving simulator. Within this virtual environment, subjects were placed within the driver's seat of a vehicle on a closed-loop circuit. Subjects were provided with a virtual vehicle interior including a graphical representation of the vehicle's current speed. The placement of this indicator was consistent with that of traditional speedometer placement. The closed-loop circuit consisted of four turns which were created with the assistance of a gray surface to simulate the road and a green surface to simulate the grass.

The secondary display provided the experiment administer with a graphical user interface supporting the haptic-enabled rotary control knob (see Figure 3). This screen utilizes a vertical progress bar to correlate $360^{\circ}$ of knob rotation to adjust the window height from $0 \%$ height to $100 \%$ height. Additionally, this screen provides the experiment administer with a numerical representation of the window height, an option to select which situation to run, and an option to reset the window height to $0 \%$ height.

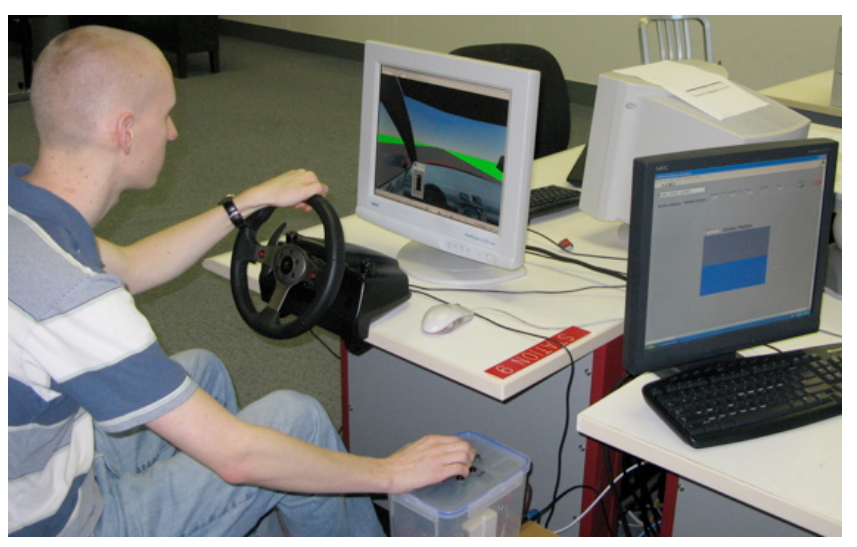

Figure 2. Subject sitting within the driver's seat of the driving simulator while making window height adjustments. 
Using the aforementioned setup, a single study was conducted among 8 healthy drivers ( 6 males and 2 females) between the ages of 19 and 38. This study focused on examining the accuracy at which a subject could adjust the height of the window by relying solely on force-feedback provided by a haptic-enabled rotary control knob. This study was completed as described in the following paragraphs:

During this study, haptic feedback was generated by means of a "constant force" exerting approximately $1.34 \pm 0.02 \mathrm{~N}$ on the subject's hand. Utilizing this force, subjects were exposed to two situations:

I. Window adjustment without intermediate haptic feedback.

II. Window adjustment with varying durations of intermediate haptic feedback.

Within each situation, $100 \%$ height was indicated by a stop restricting rotation beyond this point. Case I provided the subject with no haptic feedback as window height was adjusted. Case II provided subjects with haptic feedback at $25 \pm 0.4 \%, 50 \pm 3.125 \%$, and $75 \pm 6.25 \%$ of the maximum window height $(100 \%)$ as window height was adjusted. Consequently, subjects experience the "constant force" between the following window heights: $24.6 \%$ to $25.4 \%, 46.875 \%$ to $53.125 \%$, and $68.75 \%$ to $81.25 \%$. Increasing durations of haptic feedback are utilized to differentiate between the three window height levels $(25 \%, 50 \%$ and $75 \%)$. Naturally, the duration of haptic feedback experienced by the subject increases as the window height increases. This is done to allow users to better estimate window height during adjustment.

Using these conditions, subjects were asked to adjust the height of the window to a target window height (in percentage form) while driving in the simulator. Window adjustments for 10 window heights were requested in random order for situation 1 and then situation 2 . The target window heights requested were as follows: $15 \%, 25 \%, 35 \%, 45 \%, 50 \%, 60 \%, 70 \%, 75 \%, 80 \%$, and $90 \%$. While the experiment was in progress, the subject was required to maintain their speed within a specified range, stay within the virtual road, and complete the window adjustment within 5 seconds. If either of these conditions were violated, the window

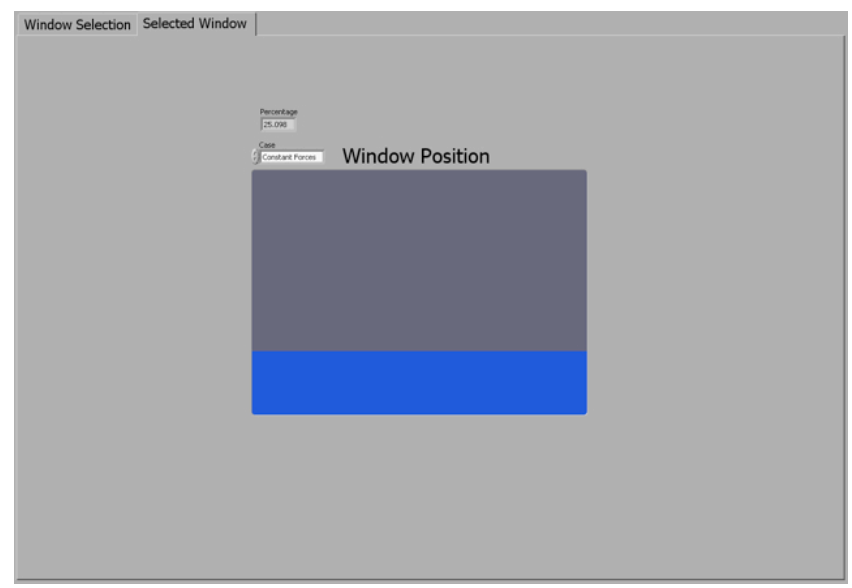

Figure 3. Window Position screen utilized within the study to monitor the window height (in graphical and percentage form) and current situation being run. adjustment was disregarded and the target window height was requested again later in the series of trials. To remove reliance on visual feedback, the window adjustment task display was positioned such that only the experiment administer could view it. After each trial the experiment administer recorded the adjusted window height and reset the window height to $0 \%$. This procedure was completed for all trials comprising both situations.

\section{RESULTS AND DISCUSSION}

After gathering data, the adjusted window heights recorded during each trial were compared to the target window heights for that specific condition. The average percentage difference between the adjusted window height and target window height for each condition were then computed (see Figure 4). Examining this figure, results demonstrate that providing a subject with varying intermediate haptic feedback improves accuracy when adjusting window height. Specifically, providing the subject with varying intermediate forces resulted in a $4.03 \%$ difference from the target height while the situation without resulted in a $13.89 \%$ difference from the target height. More importantly, results suggest that it is possible to remove reliance on visual feedback when adjusting window height while maintaining adjustment accuracy.

To determine the statistical significance of this data, a one-way repeated measures ANOVA was conducted. The results confirm the hypothesis, $\mathrm{H}_{1}$, in favor over the null hypothesis $(\mathrm{F}(1,7)=$ 287.0625; $\mathrm{p}=0.00016382)$. Accordingly, these results successfully demonstrate that the use of varying intermediate haptic feedback allows subjects to accurately adjust window height utilizing haptic feedback alone.

\section{CONCLUSIONS AND FUTURE WORK}

The objective of this paper was to investigate the possibility of an accuracy improvement resulting from providing a driver with varying intermediate haptic feedback when adjusting window height. This investigation was conducted to examine the ability of a haptic-enabled rotary control knob to eliminate a driver's

Percentage Difference from Target Height

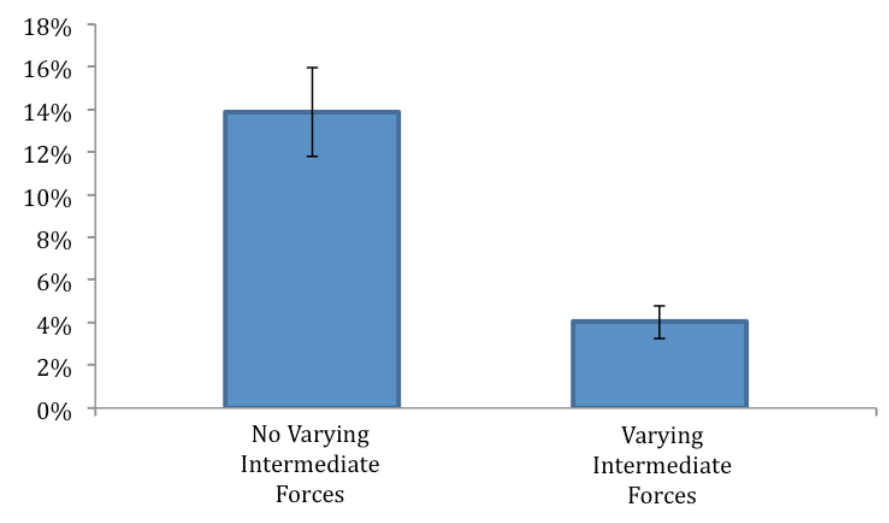

Figure 4. Bar graph depicting the percentage difference between the adjusted window height and target window height for each condition. Condition $I$ is represented by the "No Varying Intermediate Forces" bar. Condition II is represented by the "Varying Intermediate Forces" bar. Exact values are as follows; Condition $I=\mathbf{1 3 . 8 9} \%$ and Condition $I I=\mathbf{4 . 0 3} \%$. 
reliance on visual feedback when adjusting window height. The impact of auditory feedback has been overlooked to focus solely on the ability of varying intermediate haptic feedback to indicate window height during adjustment. More importantly, this investigation was conducted for the purposes of increasing driving safety by reducing driver distractions and improving driving task and window adjustment task performance.

This investigation consisted of examining an alternate approach to means of providing the driver with feedback indicating window height. As opposed to visual feedback, this investigation relied upon the use of haptic feedback to indicate window height. This was accomplished through the examination of two situations: with varying intermediate haptic feedback and without varying intermediate haptic feedback. Additionally, an alternate approach to providing drivers with window adjustment hardware was examined. As opposed to the conventional series of 4 push buttons found on the driver's side door, this investigation examined the use of a single control point centrally located within the vehicle.

Upon examining the results, it is evident that providing a subject with varying intermediate haptic feedback allows for more accurate window height adjustment. This suggests that the utilization of such a haptic-enabled rotary control knob has the ability to eliminate driver reliance on visual feedback when adjusting window height. Consequently, the safety and performance benefits experienced as a result of utilizing haptic feedback additionally applies to the task of vehicular window adjustment.

To support the reliability of these results, future work must be conducted utilizing the system within a real driving situation. In addition to eliminating differences in driving behavior, this also acts to determine the performance differences that may occur as a result of a driver also relying on auditory feedback when making window adjustments. Furthermore, the benefits of implementing a complete window control system supported by a haptic-enabled rotary control knob will be examined through an extensive study.

\section{ACKNOWLEDGMENTS}

This research has been performed with the assistance of equipment supplied by Kettering University in the on-site haptic laboratory. The authors would like to thank all of the subjects who participated in the study for their time and comments regarding this application.

\section{REFERENCES}

[1] Audi A8/S8 Quick Reference Guide, http://www.audiusa.com.

[2] Audi Multi Media Interface, http://www.audiusa.com.

[3] Badescu, M., Wampler, C., and Mavroidis, C. 2002. Rotary Haptic Knob for Vehicular Instrument Controls. In Proceedings of the $10^{\text {th }}$ Symp. On Haptic Interfaces For Virtual Environments \& Teleoperator Systems (Orlando, Florida, March 24-25, 2002).

[4] BMW iDrive Control System, http://www.bmw.com.

[5] Hannaford, B., Wood, L., McAffee, D. A., and Zak, H. 1991. Performance Evaluation of a Six-Axis Generalized ForceReflecting Teleoperator. In IEEE Transactions on Systems, Man, and Cybernetics (May/June 1991). Vol. 21, No. 3, pp. 620-633.

[6] Haptics for the Automotive Market - Immersion Corporation, http://www.immersion.com.

[7] Hjelm, J. 2008. Haptics in Cars. Univerisity of Tampere Seminar on Haptic Communication and Interaction in Mobile Contexts.

[8] MacLean, K. E., and Roderick, J. B. 1999. Smart Tangible Displays in the Everyday World: A Haptic Door Knob. In Proceedings of IEEE/ASME International Conference on Advanced Intelligent Mechatronics (Atlanta, Georgia, September 19-13, 1999). AIM'99.

[9] MacLean, K. E., Hasser, C. J., and Chu, L. 2001. Driving with Programmable Haptic Feedback: Design Scenarios and Contextual Evaluation, Submitted to UIST 2001.

[10] Racine, D., Holmen, J. K., Zadeh, M. H., and Thompson, M. 2010. Improving Vehicular Window Control with Haptic and Visual Feedback, In Proceedings of EuroHaptics 2010 (Amsterdam, Netherlands, June 8-10, 2010).

[11] Swindells, C., MacLean, K. E., Booth, K. S., and Meitner, M. 2007. Exploring Affective Design for Physical Controls In Proceedings of ACM Conference on Human Factors in Computing Systems (San Jose, California, USA, April 28May 3, 2007). CHI '07. Vol. 9, No. 1, pp. 933-942.

[12] Vitrani, M., Nikitczuk, J., Morel, G., and Mavroidis, C. 2006. Torque Control of Electro-Rheological Fluidic Resistive Actuators for Haptic Vehicular Instruments Control. In Transactions of the ASME Journal of Dynamic Systems, Measurement and Control. Vol. 128, No. 2, pp. 216-226. 